\title{
Resolution and image fidelty in STEM and TEM (HREM)
}

\author{
J.C.H.Spence
}

Department of Materials, Cambridge University, Cambridge, UK. CB2 3QZ. (Spence@asu.edu)

Does aberration-corrected ADF STEM have better resolution than thro-focus TEM (HREM) ? Which mode has fewer image artifacts ? First, recall that resolution cannot be defined if it depends on properties of the object (phase shifts). Only the incoherent imaging model for ADF STEM and the weak-phase object model for HREM provide a sample-independent basis for comparison -these have very limited domains of validity. (Reciprocity shows more generally how to obtain identical resolution in TEM and STEM). The advent of the aberration corrector for TEM can bring an improved point-resolution limit out to the information limit, if defocus $\square \mathrm{f}=-\left(\mathrm{C}_{\mathrm{s}} \square\right)^{1 / 2}$ with $\mathrm{C}_{\mathrm{s}}$ set electronically to $\square^{2} \square^{2} / \square[1]$. Here $\square$ is the electronic instability limit due to lens and high-voltage instabilities [2]. Setting $\mathrm{C}_{\mathrm{s}}=0$ would allow use of the projected charge-density approximation [2]. The use of the negative Scherzer focus (NCSI) mode in an aberration-corrected TEM gives bright, light atoms on a dark (sample-dependant) background [3] at about $0.12 \mathrm{~nm}$ point resolution. We now compare the theoretical basis of resolution estimation for three modes - aberration-corrected HREM, through-focus HREM, and aberration corrected STEM. For these modes, published experimental "point" resolution figures are currently about $0.08 \mathrm{~nm}$ [4], $0.078 \mathrm{~nm}$ [5], and 0.075nm [ 6] respectively. To place this in perspective, uncorrected high-voltage HREM has achieved a pointresolution of $0.1 \mathrm{~nm}$ [7], while an information limit of $0.062 \mathrm{~nm}$ was attained by TEM in 1978 [8].

For aberration-corrected HREM in the wpo approximation, given adequate mechanical stability, the resolution d may approach the information limit $\mathrm{d}^{-1}=\mathrm{u}_{\mathrm{o}}(\square)=[2 /(\square \square \square)]^{1 / 2}$, which depends only on beam energy and $\square=\mathrm{R}^{*} \mathrm{C}_{\mathrm{c}}$, where $\mathrm{C}_{\mathrm{c}}$ is the chromatic aberration constant and $\mathrm{R}$ the RMS quadrature sum of fluctuations in high-voltage, lens power supply currents and energy spread at the electron source [2]. This applies also to the NCSI condition. Thus $\square$ can be reduced, for example, through the use of a monochromator in combination with Cs corrector, as in the Zeiss UHRTEM [4,] or by using a cold field-emitter with smaller energy spread. A Cs corrector reduces the damping due to partial spatial coherence at the expense of increased Cc, which must be considered. The transfer function for FEG coherent illumination has a different form from that for conventional sources [1].

Uncorrected through-focus image restoration schemes in HREM are also limited by $\square$ and $\mathrm{C}_{\mathrm{c}}$ (which may decrease at higher energy) and by partial spatial coherence. Sub-Angstrom resolution has been demonstrated [1,5], at the expense of computational effort and stability. (See work by Schiske, Coene, Saxton, Thust, O'Keefe, van Dyke, Kirkland, Kisielowski and others referenced in [2, 9]).

For STEM, we may distinguish three modes - bright-field, dark-field, and HAADF. HAADF collects mainly high-angle thermal diffuse scattering for Z-contrast, while dark-field collects scattered Bragg beams for diffraction contrast, but this Bragg scattering also modulates the HAADF signal [2]. To minimize this, Howie suggested an inner cutoff for HAADF at the point where the d-spacing is equal to the thermal vibration amplitude (e.g. $40 \mathrm{mrad}$ ), thus suppressing diffraction contrast at lower angles, for imaging heavy catalysts on thin crystals [10]. All three modes have demonstrated high resolution [11], and the aberration-corrected STEM has revived interest in the bright-field mode by increasing the numerical aperture and hence the signal available. In all modes, lattice resolution of 
spacing $d_{h k 1}$ requires coherent illumination over an angular semiangle of at least $\square_{\square}=\square / d_{h k 1}$, causing adjacent orders to interfere (this fundamental criteria supercedes any consideration of probe "size"). Since this arrangement is equivalent, by reciprocity, to the "super-resolution" tilted illumination (or annular hollow-cone) mode in TEM [1,8] where the optic axis bisects the direct and diffracted beam, we see immediately the resolution advantage of STEM in dark-field or HAADF. With the direct beam tilted in TEM to appear near the edge of a central objective aperture and the first-order Bragg beam lying across a diameter against the other edge, resolution is improved by a factor of two at the cost of image distortion, as it is in HAADF STEM. (In practice larger scattering angles are used in STEM, but the resolution gain is preserved). Tip vibration may ultimately limit STEM resolution.

A large literature exists on image fidelity in STEM -see [2,12] for important papers by Hillyard and Silcox, Treacy and Gibson, Rez, Nellist, Jesson and Pennycook, Ade, Allen, Cowley, Fertig and Rose. To summarize: i ) For dark field STEM, Crewe's argument that images of isolated atoms are similar to dark-field HREM images, and hence non-linear in potential, can be supported [13]. ii) For HAADF STEM one may think of the hole in the detector as an incoherent source - making this three times larger than the objective aperture will minimize interference between adjacent atomic columns and thickness fringes, but not interference along atom strings. Then, for a limited range of thickness the image becomes a convolution between the probe intensity and an inelastic (eg phonon) scattering cross-section located on atom strings. The image is then a map of the absorption potential, which saturates with thickness. Multiphonon scattering is equivalent to Rutherford scattering from the nucleus in an Einstein model, and so goes as approximately as $Z^{2}$. iii) The BF STEM image is simply related to the corresponding HREM image by reciprocity. Since total elastic scattering is independent of probe position, $\mathrm{I}_{\mathrm{STEM}}{ }^{\mathrm{DF}}(\mathrm{r})=1-\mathrm{I}_{\mathrm{STEM}}{ }^{\mathrm{BF}}(\mathrm{r})=1-\mathrm{I}_{\mathrm{HREM}}{ }^{\mathrm{BF}}(\mathrm{r})$. The safest procedure for STEM and HREM is to match simulated images of any known crystalline structure near a defect of interest first, using available software [14]. This also provides the best way to measure resolution [1], which otherwise allows additional opportunities for mischief. (Young's fringes for HREM, Peak widths or Fourier analysis of image intensity for STEM). Measurements based on images of isolated atoms must be treated with great caution. Diffractive imaging offers an interesting alternative [15].

References

[1] M. O'Keeffe Proc . MSA Suppl 2, p.1036 (2000) and O'Keefe and Allard. Proc MSA (2004).

[2] J.C.H.Spence. High Resolution Electron Microscopy, Third Edn (2003). O.U.P.

[3] C.L. Jia and K. Urban. Science 303, (2004) p.2001

[4] C. Zeiss UHRTEM with monochromator and CEOS Cs corrector. See www.smt.zeiss.com/nts

[5] C. Kisielowski et al. Ultramic. 89, (2001) 243 . Ayache et al Interface Science 2005 In press.

[6] P. Batson et al Nature 418, (2002) p.617 also Nellist et al Science 305, (2004) 1741 .

[7] H. Ichinose et al., J. Elec. Micros. 48, 887 (1999), R. Schweinfest et al Phil Mag 81, (2001) 927.

[8] T. Matsuda et al. Jap J.Appl Phys. 17, (1978) 2073.

[9] A. Thust et al Ultramic 64, 249 (1996), O. Saxton Ultramic. 55, (1994) 171.

[10] A. Howie (1979). J. Micros. 117, (1979) 11.

[11] A. Crewe et. al. Science 168, (1970) 1338 . J. Cowley Bull Mat Sci. 6,(1984) 477 , S. Pennycook et al., Nature 336, (1988) 565.

[12 ] S. Findlay, L.Allen,M.Oxley, C.Rossouw. Ultramic 96, p.65 (2003) give the modern approach.

[13] J. Cowley Ultramic. 2, (1976) 3 .

[14] E. Kirkland Advanced ComputingElectron Microscopy, Plenum,1998. http://emaps.mrl.uiuc.edu/ [15] J. Zuo et al. Science 300, (2003) 1419. Thanks to EPSRC, C.Kisielowski, H.Rose, M. O'Keefe. 